\title{
Dupilumab Treatment Provides Sustained Improvements Over 2 Years in Symptoms and Quality of Life in Adults with Atopic Dermatitis
}

\author{
Gil Yosipovitch (D) - Marjolein de Bruin-Weller (D) - April Armstrong (D) · \\ Jashin J. Wu (D) · Pedro Herranz (D) · Diamant Thaçi (D) • \\ Dimittri Delevry (D) - Gaëlle Bégo-Le Bagousse · Raymond Zhang (D) • \\ Brad Shumel (D) A Ana B. Rossi (D) · Jingdong Chao
}

Received: September 16, 2021 / Accepted: October 4, 2021 / Published online: October 29, 2021

(C) The Author(s) 2021

\section{ABSTRACT}

Introduction: Atopic dermatitis (AD) can have a profound negative impact on the quality of life (QoL) of patients. We analyzed the longterm changes in $\mathrm{AD}$ symptoms, QoL, and patient assessment of treatment effect in adults with moderate-to-severe $\mathrm{AD}$ treated for 2 years with dupilumab.

Methods: LIBERTY AD OLE (NCT01949311) is a multicenter, open-label extension (OLE) study in adults with moderate-to-severe $\mathrm{AD}$ who

Supplementary Information The online version contains supplementary material available at https:// doi.org/10.1007/s13555-021-00630-9.

G. Yosipovitch

Department of Dermatology and Itch Center, University of Miami Miller School of Medicine, Miami, FL, USA

M. de Bruin-Weller

National Expertise Center of Atopic Dermatitis, Department of Dermatology and Allergology, University Medical Center Utrecht, Utrecht, The Netherlands

A. Armstrong

Department of Dermatology, Keck School of

Medicine at USC, University of Southern California, Los Angeles, CA, USA

J. J. Wu

Dermatology Research and Education Foundation, Irvine, CA, USA previously participated in dupilumab clinical trials (parent studies). Patients received dupilumab $300 \mathrm{mg}$ weekly. Patient-Oriented Eczema Measure (POEM), Dermatology Life Quality Index (DLQI), EQ-5D-3L, and the Patient Global Assessment of Treatment Effect (PGATE) were assessed at weeks 48 and 100 .

Results: A total of 2677 patients were included in the OLE, and 1028 completed week 100. By weeks 48 and 100, 94.1\% and $95.6 \%$ of patients achieved $\mathrm{a} \geq 4$-point change in POEM from the parent study baseline (PSBL), respectively, and 93.3\% and $93.4 \%$ of patients had achieved a $\geq 4$-point change in DLQI from PSBL, respectively. At week 100, 35.1\% of patients had a POEM score $\leq 2 \quad$ (AD clear/almost clear)

\section{P. Herranz}

Department of Dermatology, La Paz University

Hospital, Madrid, Spain

D. Thaçi

Institute and Comprehensive Center for

Inflammation Medicine, University of Lübeck,

Lübeck, Germany

D. Delevry · R. Zhang · B. Shumel · J. Chao ( $\square)$

Regeneron Pharmaceuticals, Inc., 777 Old Saw Mill

River Rd, Tarrytown, NY 10591, USA

e-mail: jingdong.chao@regeneron.com

G. B.-L. Bagousse

Sanofi, Chilly Mazarin, France

A. B. Rossi

Sanofi Genzyme, Cambridge, MA, USA 
compared with $0.1 \%$ at PSBL, and $49.9 \%$ had a DLQI score of 0 or 1 (no effect at all on patient's life) compared with $1.5 \%$ at PSBL. At week 100 , 74.5-97.3\% of patients reported no effect of $\mathrm{AD}$ on the individual EQ-5D-3L domains, and 93.8\% rated the effect of dupilumab treatment as "excellent," "very good," or "good" according to PGATE.

Conclusion: In adults with moderate-to-severe $\mathrm{AD}$, dupilumab treatment over 2 years resulted in sustained improvements in patient-reported symptoms and QoL and a favorable patient perception of treatment effect.

Trial registration: ClinicalTrials.gov Identifier: NCT01949311.

\section{PLAIN LANGUAGE SUMMARY}

Atopic dermatitis is a common skin disease that causes scaly, itchy skin. It can have a profoundly negative effect on a patient's quality of life (QoL). In short-term clinical trials, dupilumab treatment resulted in significant improvements in signs and symptoms of atopic dermatitis, and in the QoL reported by patients, together with acceptable safety. In this study, adults with moderate-to-severe atopic dermatitis who had completed one of the short-term clinical trials continued dupilumab treatment, including those who had taken placebo. This study allowed researchers to continue to evaluate how dupilumab worked in the long term, including its impact on patient-reported outcomes, which measure the success of treatment from the patient's own perspective. The results were evaluated at approximately 1 and 2 years of this open-label extension study and were compared with the period just before the patient was first treated with dupilumab so that the effect of dupilumab could be seen. At approximately 1 and 2 years, most patients had achieved clinically meaningful improvements in two measures: Patient Oriented Eczema Measure, a tool used by patients to self-report the severity of their symptoms, and Dermatology Life Quality Index, which allows patients to report the effect of the disease on their QoL. Additionally, in this open-label extension study, most patients described their experience of being treated with dupilumab as "excellent," "very good," or "good" using the Patient Global Assessment of Treatment Effect questionnaire. Dupilumab treatment resulted in sustained improvements in atopic dermatitis and was regarded favorably by patients.

Keywords: Adult; Atopic dermatitis; Clinical trial; Dermatology Life Quality Index; Dupilumab; Efficacy; EQ-5D; Long term; Patient-oriented eczema measure; Patientreported outcomes

\section{Key Summary Points}

\section{Why carry out this study?}

Atopic dermatitis (AD) can have a profound negative impact on the quality of life (QoL) of patients.

We analyzed the long-term changes in symptoms, QoL, and patient assessment of treatment effect in adults with moderate-to-severe AD treated for 2 years with dupilumab.

\section{What was learned from the study?}

In adults with moderate-to-severe AD, dupilumab treatment over 2 years resulted in sustained improvements in patientreported symptoms and QoL and a favorable patient perception of treatment effect.

\section{DIGITAL FEATURES}

This article is published with digital features, including video abstract, to facilitate understanding of the article. To view digital features for this article go to https://doi.org/10.6084/ m9.figshare.16720783. 


\section{INTRODUCTION}

Atopic dermatitis (AD), a chronic inflammatory skin disease characterized by intense itch and recurring eczematous lesions, can have a profound negative impact on the quality of life (QoL) of patients [1-3].

Dupilumab is a fully human monoclonal antibody that blocks the shared receptor subunit for IL-4 and IL-13, thus inhibiting signaling of these key and central inflammatory cytokines in $\mathrm{AD}$ pathophysiology. Dupilumab is approved for patients with type 2 inflammatory diseases, including $\mathrm{AD}$, asthma, and chronic rhinosinusitis with nasal polyps $[4,5]$. In phase 3 trials in adults and adolescents with moderate-tosevere $A D$ and in children with severe $A D$, dupilumab was shown to significantly improve clinical signs, symptoms, and QoL with an acceptable safety profile [6-10]. In an ongoing, open-label extension (OLE) study in adults with moderate-to-severe AD (LIBERTY AD OLE; NCT01949311), dupilumab has also demonstrated sustained efficacy with an acceptable safety profile for up to 3 years [11].

In this study we report the long-term changes in symptoms and QoL as well as patient assessment of treatment effect in adults with moderate-to-severe $\mathrm{AD}$ treated for 2 years with dupilumab in an OLE study (LIBERTY AD OLE). A short author video summarizing the key findings of this study can be found in the online version of the manuscript.

\section{METHODS}

\section{Study Design}

LIBERTY AD OLE (NCT01949311) is an ongoing phase 3, multicenter, OLE clinical trial in adults with moderate-to-severe $\mathrm{AD}$ who previously participated in phase 1-3 clinical trials of dupilumab. The study design has been previously reported [11].

Briefly, patients with moderate-to-severe AD were eligible for inclusion if they had adequately completed the required assessments of the parent phase 1-3 dupilumab studies. Patients treated with dupilumab in the parent studies (prior dupilumab treated) as well as patients who received placebo in the parent studies (dupilumab treatment-naïve) were included. Patients were excluded if they had experienced dupilumab-related adverse events (AEs) or serious AEs (SAEs) leading to discontinuation from the parent studies. Treatment consisted initially (October 2013) of subcutaneous dupilumab $200 \mathrm{mg}$ weekly (qw), which was revised following a protocol amendment (December 2013) to $300 \mathrm{mg}$ qw. Concomitant topical corticosteroids (TCS) were allowed without restriction.

The study was conducted in 28 countries in accordance with the Declaration of Helsinki, the International Council for Harmonisation Good Clinical Practice guideline, and applicable regulatory requirements. The local institutional review board or ethics committee at each study center oversaw trial conduct and documentation. All patients provided written informed consent before participating in the trial.

\section{Patient-Reported Outcomes}

AD symptoms and their effect on QoL can be evaluated using multiple validated patient-reported outcome (PRO) measures, including both disease-specific and general health status PROs. Among these, Patient-Oriented Eczema Measure (POEM) can be used to evaluate the frequency of seven $\mathrm{AD}$ signs and symptoms during the past week, including skin manifestations, itch, and sleep disturbance [12]. The minimal clinically important difference (MCID) established for POEM is a reduction of 3.4 points [13]. The burden of skin disease in adult patients can be assessed using the Dermatology Life Quality Index (DLQI) [14, 15]. The MCID established for DLQI in inflammatory skin diseases is a 4-point reduction [16]. The Patient Global Assessment of Treatment Effect (PGATE) instrument measures patient-reported global assessment of treatment effect by asking patients: "How would you rate the way your eczema responded to the study medication?" PGATE rating was based on a 5-point scale ranging from "Poor" to "Excellent." Additionally, the EQ-5D-3L questionnaire, a generic 
health-related QoL instrument, measures overall health-related QoL of patients in five dimensions (mobility, self-care, usual activities, pain/discomfort, anxiety/depression). Each dimension has three levels of severity: no problem (1), some problems (2), and severe problems (3). The EQ-5D-3L consists of two components: a health state profile and a visual analog scale (VAS), with which a patient captures their current QoL state on a scale from 0 (worst imaginable health) to 100 (best imaginable health) [17].

The primary objective of this OLE study was to assess the long-term safety of dupilumab. Additional outcomes, particularly PROs, were assessed through week 100 only as a result of a 2016 protocol amendment in which these assessments were removed to reduce the burden to investigators and patients.

For the purpose of this study, two baselines were defined: parent study baseline (PSBL) and OLE baseline (the point of entry into the current OLE study). POEM, DLQI, and EQ-5D-3L assessments were performed at both parent study and OLE baselines and at weeks 48 and 100. PGATE was collected at weeks 48 and 100.

Endpoints reported here include proportions of patients who achieved a change in POEM $\geq 4$ points from baseline of parent study at weeks 48 and 100 and proportion of patients who maintained $\mathrm{a} \geq 4$-point change in POEM at week 100 , among those who had achieved the change at week 48, and distribution of patients in categorical severities of POEM (0-2: clear/almost clear; 3-7: mild; 8-16: moderate; 17-24: severe; 25-28: very severe) [12] at parent study and OLE baselines and at week 100 .

Endpoints also included proportions of patients who achieved a change in DLQI $\geq 4$ points from baseline of parent study at weeks 48 and 100 and proportion of patients who maintained $\mathrm{a} \geq 4$-point change in DLQI at week 100, among those who had achieved the change at week 48, and distribution of patients in categorical severities of DLQI (0-1: no effect at all on patient's life; 2-5: small effect; 6-10: moderate effect; $11-20$ very large effect; $21-30$ extremely large effect) [18] at parent study and OLE baselines and at week 100. Proportions of patients reporting "None" (i.e., no effect of AD on that domain) for the five individual domains of EQ-5D-3L at week 100 and PGATE with dupilumab at weeks 48 and 100 were also evaluated.

\section{Analysis}

All analyses were carried out in the safety analysis set (SAF), which included all patients in LIBERTY AD OLE who received at least one dose of dupilumab and completed the week 100 visit. Endpoints were analyzed descriptively using all observed data. There was no imputation for missing data.

For continuous variables, descriptive statistics include the number of patients reflected in the calculation $(n)$, mean, and standard deviation (SD). For categorical or ordinal data, frequencies and percentages of observed values are given for each category. No formal statistical hypotheses were tested.

\section{RESULTS}

\section{Patients}

A total of 2677 patients were included in LIBERTY AD OLE and received study treatment. At the time of the interim database lock, $82.4 \%$ of patients $(2207 / 2677)$ had completed up to week $52,1028(38.4 \%)$ up to week 100 , and 347 $(13.0 \%)$ up to week 148 [11]. Of the 1325 $(49.5 \%)$ patients who withdrew from the study, most $(807$ [30.1\%]) did so because of per-protocol study termination by the sponsor upon regulatory approval/commercial availability of the drug and 57 (4.3\%) because of lack of efficacy [11]. The patient disposition, baseline demographics, and disease characteristics for this patient population in LIBERTY AD OLE have been previously reported, along with efficacy, and overall safety outcomes up to 3 years [11].

At the parent study and OLE baselines, patients had a mean age of approximately 39 years (Table 1). Mean DLQI and POEM scores were higher at the PSBL compared with the OLE baseline, and mean EQ-5D VAS score was higher 
at the OLE baseline, reflecting improvement in dupilumab-treated patients in the parent study (Table 1).

\section{POEM}

At weeks 48 and 100, 94.1\% and $95.6 \%$ of patients had achieved $\mathrm{a} \geq 4$-point reduction in POEM from the PSBL, respectively, and 91.5\% of patients achieved this change at both weeks 48 and 100 (Table 2). Almost all (97.2\%) of the patients who achieved $\mathrm{a} \geq 4$-point change in POEM at week 48 maintained the change at week 100.

At week 100, 35.1\% of patients had a POEM score of $\leq 2$ (AD clear/almost clear) compared with $0.1 \%$ at PSBL (Fig. 1a).

\section{QoL Assessments}

By weeks 48 and 100, 93.3\% and 93.4\% of patients had achieved $\mathrm{a} \geq 4$-point reduction in DLQI from the PSBL, respectively, and 90.3\% achieved this change at both weeks 48 and 100 (Table 2). Almost all (96.8\%) of the patients who achieved $\mathrm{a} \geq 4$-point change in DLQI at week 48 maintained the change at week 100. At week 100 , half of patients $(49.9 \%)$ had a DLQI score of 0 or 1 (no effect at all on patient's life) compared with $1.5 \%$ at PSBL (Fig. 1b).

At week 100, for each of the five individual domains of the EQ-5D-3L (mobility, self-care, usual activities, pain/discomfort, anxiety/depression), most patients (range 74.5-97.3\%) reported "None" (i.e., no effect of $\mathrm{AD}$ on that domain) (Table 2).

\section{PGATE}

At weeks 48 and 100, almost all patients (91.7 and $93.8 \%$, respectively) reported dupilumab treatment effect was "good," very good," or "excellent" according to the PGATE scale (Fig. 2).

Table 1 Baseline demographics and clinical characteristics

\begin{tabular}{lcc}
\hline Characteristic & \multicolumn{2}{l}{ Dupilumab 300 mg qw $(\boldsymbol{N}=\mathbf{2 6 7 7})$} \\
\cline { 2 - 3 } & Parent study baseline & OLE study baseline \\
\hline Age, mean (SD), years & $39.1(13.47)$ & $39.2(13.42)$ \\
Male, number (\%) & $1453(61.4)$ & $1611(60.2)$ \\
Race, number (\%) & & \\
White & $1703(72.0)$ & $1936(72.3)$ \\
Black or African American & $109(4.6)$ & $147(5.5)$ \\
Asian & $512(21.6)$ & $541(20.2)$ \\
Other & $27(1.1)$ & $33(1.2)$ \\
Not reported & $15(0.6)$ & $20(0.7)$ \\
Duration of AD, mean (SD), years & $29.0(14.8)$ & $29.9(14.8)$ \\
POEM (range 0-28), mean (SD) & $20.4(5.91)$ & $14.7(8.00)$ \\
DLQI score (range 0-30), mean (SD) & $14.7(7.39)$ & $8.5(7.11)$ \\
EQ-5D VAS (range 0-100), mean (SD) & $55.1(23.66)$ & $66.2(22.03)$ \\
\hline
\end{tabular}

$A D$ atopic dermatitis, $D L Q I$ Dermatology Life Quality Index, OLE open-label extension, POEM Patient-Oriented Eczema Measure, $q w$ weekly, $S D$ standard deviation, $V A S$ visual analog scale 
Table 2 Study outcomes: POEM, DLQI, and EQ-5D-3L

Dupilumab $300 \mathrm{mg}$ qw

$(N=2677)$

Patients achieving POEM change of $\geq 4$ points from PSBL, $\mathrm{n} / \mathrm{N} 1^{\mathrm{a}}(\%)$

Week 48

$723 / 768(94.1)$

Week 100

$734 / 768(95.6)$

Weeks 48 and 100

$703 / 768(91.5)$

Patients maintaining POEM change of $\geq 4$ points at week 100, of patients achieving POEM 703/723 (97.2) change of $\geq 4$ points at week $48, \mathrm{n} / \mathrm{N} 2^{\mathrm{b}}(\%)$

Patients achieving DLQI change of $\geq 4$ points from PBSL, $\mathrm{n} / \mathrm{N1}^{\mathrm{a}}(\%)$

Week 48

654/701 (93.3)

Week 100

655/701 (93.4)

Weeks 48 and 100

633/701 (90.3)

Patients maintaining DLQI change of $\geq 4$ points at week 100 , of patients achieving DLQI change of $\geq 4$ points at week $48, \mathrm{n} / \mathrm{N} 2^{\mathrm{b}}(\%)$

$633 / 654(96.8)$

Patients with "None" for each EQ-5D-3L domain, n/N1" (\%)

Mobility

PSBL

$599 / 750$

(79.9)

Self-care

$626 / 750$

Usual activities

Pain/discomfort

Anxiety/depression

DLQI Dermatology Life Quality Index, OLE open-label extension, POEM Patient-Oriented Eczema Measure, PSBL parent study baseline, $q w$ weekly, $S D$ standard deviation

${ }^{a}$ For POEM and DLQI, N1 are the patients who had PSBL POEM $\geq 4$ or DLQI $\geq 4$ (as relevant to the outcome specified) and OLE study baseline, week 48, and week 100 assessments

b $\mathrm{N} 2$ are the patients who had achieved a change of POEM $\geq 4$ or DLQI $\geq 4$ at week 48 based on the N1

c For EQ-5D-3L, N1 are the patients with PSBL and week 100 assessments

\section{DISCUSSION}

In this long-term OLE study in adults with moderate-to-severe $\mathrm{AD}$, almost all patients treated with dupilumab for 100 weeks achieved clinically relevant benefits from the reduction of symptoms and improvement in QoL, with the majority achieving mild disease or clear skin. Over $90 \%$ of patients rated the effect of treatment as "good," "very good," or "excellent."

A prior analysis of this OLE study found that dupilumab treatment for up to 3 years resulted in sustained improvements in $\mathrm{AD}$ signs and 
a

Severity categories for POEM

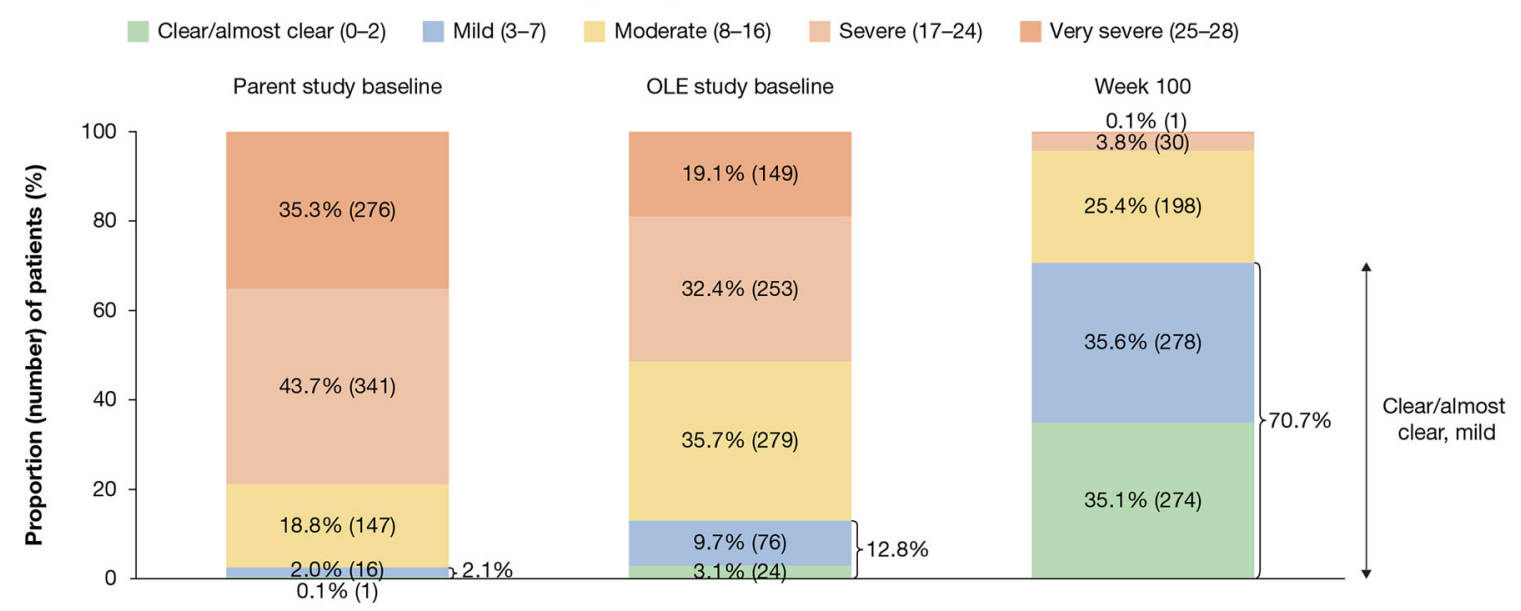

b

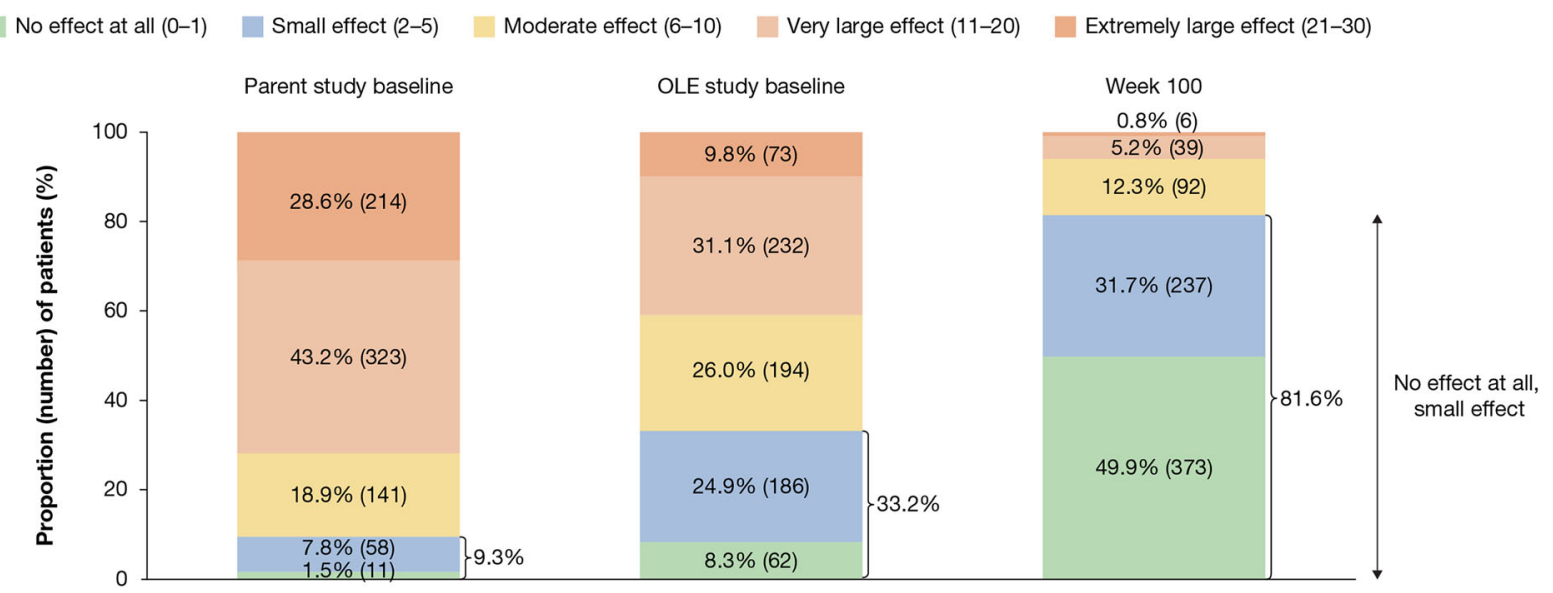

Fig. 1 Distribution of patients in categorical severities for $\mathbf{a}$ POEM and $\mathbf{b}$ DLQI at parent and OLE study baselines and at week 100. Analyses include patients with parent study baseline, OLE study baseline, and week 100 assessments. DLQI Dermatology Life Quality Index,
OLE open-label extension, POEM Patient-Oriented Eczema Measure

$\geq 50 \%$ reduction in EASI from baseline by week $100,91.3 \%$ achieved $\mathrm{a} \geq 75 \%$ reduction, and $72.8 \%$ a $\geq 90 \%$ reduction. Itch, a key patientreported symptom impacting QoL, decreased from PSBL to week 100 as measured by mean percent change in weekly pruritus Numerical Rating Scale $(-65.7 \%)$, and $79.1 \%$ of patients achieved $\mathrm{a} \geq 3$-point reduction in weekly pruritus Numerical Rating Scale from baseline [11]. These improvements in itch, as well as in other $\mathrm{AD}$ signs and symptoms reported previously [11], correspond with the improvements observed in PROs reported here. Correlations 


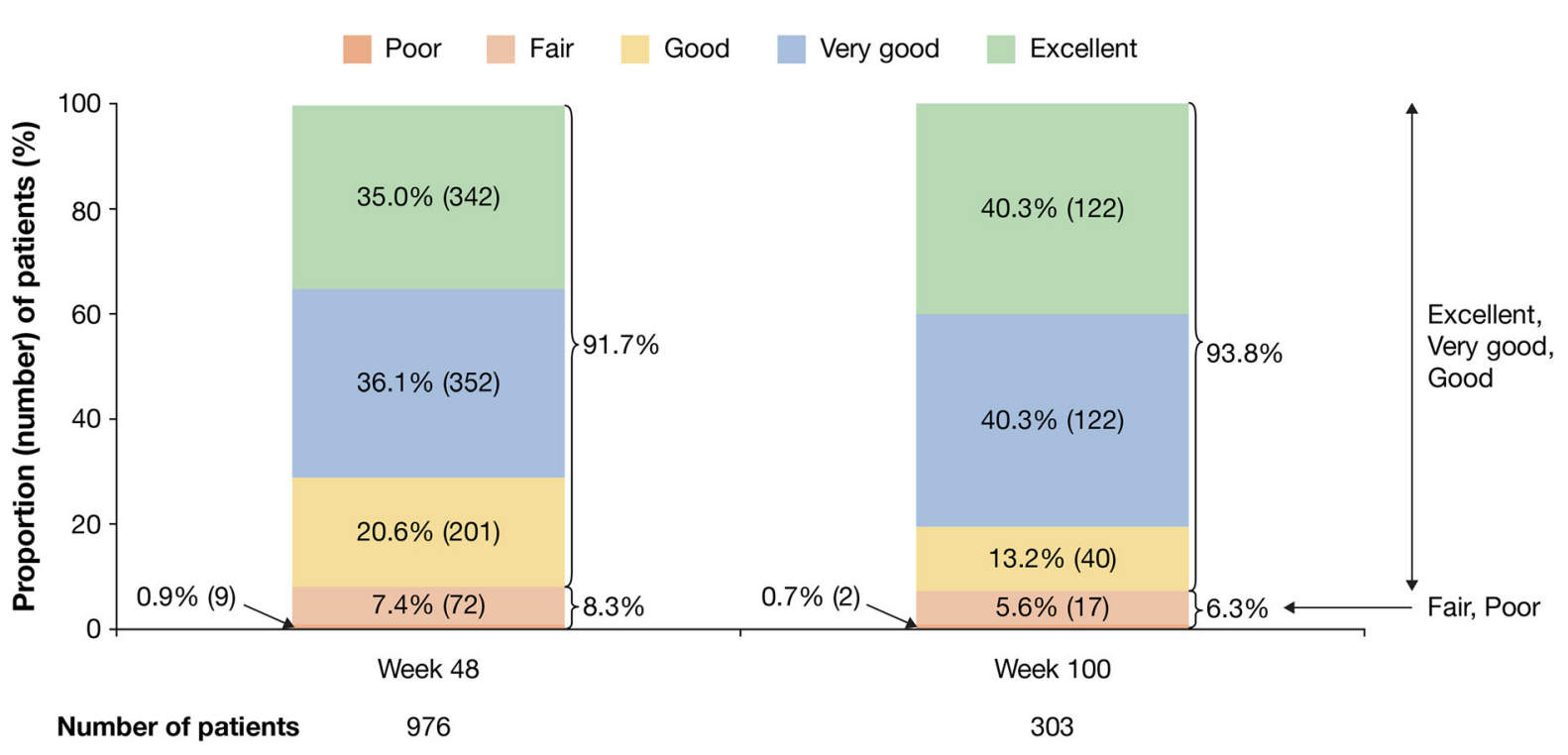

Fig. 2 Proportion of patients reporting the individual PGATE responses at weeks 48 and 100. Week 48 and week 100 analyses include patients with assessments at

and associations between improvements in clinical and PROs in adults with $\mathrm{AD}$ treated with dupilumab have been reported elsewhere $[19,20]$.

Patient perception of relief from overall $\mathrm{AD}$ disease burden is reflected by multidimensional PROs. In this analysis, most patients receiving dupilumab showed substantial improvements in the PROs POEM, DLQI, and EQ-5D-3L and favorably assessed the effect of dupilumab treatment.

Of note, adults received dupilumab $300 \mathrm{mg}$ qw in the OLE study, while the currently approved dose regimen for adults is $300 \mathrm{mg}$ every 2 weeks. Safety and efficacy were generally consistent with controlled studies [6-8]. The weekly dupilumab dosage regimen was selected for this OLE study to increase the likelihood of identifying any safety signals and to generate safety data to support both weekly and every other week regimens. The safety results confirmed an acceptable safety profile with no increase over time in rates of treatment-related AEs or SAEs (per 100 patient years) [11].

The strengths of this study include the large patient population in an international setting and long-term study duration. Limitations include the open-label study design and lack of

week 48 and week 100, respectively. PGATE Patient Global Assessment of Treatment Effect

a control arm. Additionally, fewer patients were available at later time points because of the timing of the analysis and the required withdrawal of patients upon regulatory approval of dupilumab in the individual countries in which the patients were enrolled [11].

\section{CONCLUSIONS}

In adults with moderate-to-severe $\mathrm{AD}$, dupilumab treatment over 2 years resulted in sustained improvements in patient-reported symptoms and QoL and a favorable patient perception of treatment effect.

\section{ACKNOWLEDGEMENTS}

We thank the patients and investigators who participated in the study, and the publication managers of Sanofi and Regeneron Pharmaceuticals, Inc.

Funding. This research was sponsored by Sanofi and Regeneron Pharmaceuticals, Inc. The study sponsors participated in the study design; collection, analysis, and interpretation of the 
data; writing of the report; and the decision to submit the article for publication. The study sponsors also funded the journal's Rapid Service Fee.

Authorship. All named authors meet the International Committee of Medical Journal Editors (ICMJE) criteria for authorship for this article, take responsibility for the integrity of the work as a whole, and have given their approval for this version to be published.

Author Contributions. All authors contributed to manuscript concept and design. MdBW and DT acquired data. RZ conducted the statistical analyses on the data. All authors interpreted the data, provided critical feedback on the manuscript, approved the final manuscript for submission, and are accountable for the accuracy and integrity of the manuscript.

Disclosures. Gil Yosipovitch is an advisory board member for Bellus Health, Eli Lilly, Galderma, GSK, Kiniksa Pharmaceuticals, LEO Pharma, Novartis, Pfizer, Regeneron Pharmaceuticals, Inc., Sanofi, and Trevi Therapeutics; has received grants/research funding from Kiniksa Pharmaceuticals, LEO Pharma, Novartis, Pfizer, and Sun Pharma; and is an investigator for Regeneron Pharmaceuticals, Inc. and Sanofi. Marjolein de Bruin-Weller is a consultant, advisory board member, and/or speaker for AbbVie, Almirall, Eli Lilly, Galderma, Janssen, LEO Pharma, Pfizer, Regeneron Pharmaceuticals, Inc., Sanofi Genzyme, and UCB. April Armstrong is an employee of the University of Southern California and an investigator and/or consultant for AbbVie, BMS, Dermavant, Dermira, Janssen, Eli Lilly, LEO Pharma, Modernizing Medicine, Ortho Dermatologics, Regeneron Pharmaceuticals, Inc., Sanofi Genzyme, Science 37, and UCB. Jashin J. Wu is an investigator for AbbVie, Amgen, Eli Lilly, Janssen, and Novartis; a consultant for AbbVie, Almirall, Amgen, Arcutis, Aristea Therapeutics, Boehringer Ingelheim, BMS, Dermavant, Dr. Reddy's Laboratories, Eli Lilly, Galderma, Janssen, LEO Pharma, Mindera, Novartis, Regeneron Pharmaceuticals, Inc., Sanofi Genzyme, Solius, Sun Pharmaceutical, UCB, Valeant, and Zerigo
Health; and a speaker for AbbVie, Amgen, Novartis, Regeneron Pharmaceuticals, Inc., Sanofi Genzyme, Sun Pharmaceutical, UCB, and Valeant. Pedro Herranz is a principal investigator, advisory board member, and consultant for AbbVie, Celgene, Janssen, Novartis, Pfizer, Regeneron Pharmaceuticals, Inc., and Sanofi Genzyme. Diamant Thaçi is a consultant, advisory board member, and/or investigator for AbbVie, Almirall, Amgen, Beiersdorf, BMS, Boehringer Ingelheim, Eli Lilly, Galapagos, Janssen-Cilag, LEO Pharma, MorphoSys, Novartis, Pfizer, Regeneron Pharmaceuticals, Inc., Samsung, Sandoz, Sanofi, Sun Pharma, and UCB. Gaëlle Bégo-Le Bagousse is an employee of Sanofi and may hold stock and/or stock options in the company. Ana B. Rossi is an employee of Sanofi Genzyme and may hold stock and/or stock options in the company. Dimittri Delevry, Raymond Zhang, Brad Shumel, and Jingdong Chao are employees and shareholders of Regeneron Pharmaceuticals, Inc.

Compliance with Ethics Guidelines. The study was conducted in accordance with the Declaration of Helsinki, the International Council for Harmonisation Good Clinical Practice guideline, and applicable regulatory requirements. An independent data and safety monitoring committee conducted blinded monitoring of patient safety data. The local institutional review board or ethics committee at each study center oversaw trial conduct and documentation. All patients, or their parents/guardians, provided written informed consent before participating in the trial.

Data Availability. Qualified researchers may request access to study documents (including the clinical study report, study protocol with any amendments, blank case report form, statistical analysis plan) that support the methods and findings reported in this manuscript. Individual anonymized participant data will be considered for sharing once the product and indication has been approved by major health authorities (e.g., FDA, EMA, PMDA), if there is legal authority to share the data and there is not a reasonable likelihood of 
participant re-identification. Submit requests to https://vivli.org/.

\section{Medical Writing and Editorial Assis-} tance. Medical writing/editorial assistance was provided by Carolyn Ellenberger, $\mathrm{PhD}$, of Excerpta Medica, funded by Sanofi Genzyme and Regeneron Pharmaceuticals, Inc., according to the Good Publication Practice guideline.

Open Access. This article is licensed under a Creative Commons Attribution-NonCommercial 4.0 International License, which permits any non-commercial use, sharing, adaptation, distribution and reproduction in any medium or format, as long as you give appropriate credit to the original author(s) and the source, provide a link to the Creative Commons licence, and indicate if changes were made. The images or other third party material in this article are included in the article's Creative Commons licence, unless indicated otherwise in a credit line to the material. If material is not included in the article's Creative Commons licence and your intended use is not permitted by statutory regulation or exceeds the permitted use, you will need to obtain permission directly from the copyright holder. To view a copy of this licence, visit http://creativecommons.org/licenses/by$\mathrm{nc} / 4.0 /$.

\section{REFERENCES}

1. Haeck IM, ten Berge O, van Velsen SG, de BruinWeller MS, Bruijnzeel-Koomen CA, Knol MJ. Moderate correlation between quality of life and disease activity in adult patients with atopic dermatitis. J Eur Acad Dermatol Venereol. 2012;26(2): 236-41.

2. Kim DH, Li K, Seo SJ, et al. Quality of life and disease severity are correlated in patients with atopic dermatitis. J Korean Med Sci. 2012;27(11):1327-32.

3. Weidinger S, Beck LA, Bieber T, Kabashima K, Irvine AD. Atopic dermatitis. Nat Rev Dis Primers. 2018;4(1):1.

4. European Medicines Agency. DUPIXENT® (dupilumab). Summary of product characteristics. https:// www.ema.europa.eu/en/documents/product- information/dupixent-epar-product-information_ en.pdf. Accessed June 2021.

5. US Food and Drug Administration. DUPIXENT® (dupilumab). Highlights of prescribing information. https://www.accessdata.fda.gov/drugsatfda docs/label/2020/761055s020lbl.pdf. Accessed June 2021.

6. Simpson EL, Bieber T, Guttman-Yassky E, et al. Two phase 3 trials of dupilumab versus placebo in atopic dermatitis. N Engl J Med. 2016;375(24):2335-48.

7. Blauvelt A, de Bruin-Weller M, Gooderham M, et al. Long-term management of moderate-to-severe atopic dermatitis with dupilumab and concomitant topical corticosteroids (LIBERTY AD CHRONOS): a 1-year, randomised, double-blinded, placebo-controlled, phase 3 trial. Lancet. 2017;389(10086): 2287-303.

8. de Bruin-Weller $\mathrm{M}$, Thaçi $\mathrm{D}$, Smith $\mathrm{CH}$, et al. Dupilumab with concomitant topical corticosteroid treatment in adults with atopic dermatitis with an inadequate response or intolerance to ciclosporin A or when this treatment is medically inadvisable: a placebo-controlled, randomized phase III clinical trial (LIBERTY AD CAFÉ). $\mathrm{Br} J$ Dermatol. 2018;178(5):1083-101.

9. Simpson EL, Paller AS, Siegfried EC, et al. Efficacy and safety of dupilumab in adolescents with uncontrolled moderate to severe atopic dermatitis: a phase 3 randomized clinical trial. JAMA Dermatol. 2020;156(1):44-56.

10. Paller AS, Siegfried EC, Thaçi D, et al. Efficacy and safety of dupilumab with concomitant topical corticosteroids in children 6 to 11 years old with severe atopic dermatitis: a randomized, double-blinded, placebo-controlled phase 3 trial. J Am Acad Dermatol. 2020;83(5):1282-93.

11. Beck LA, Thaçi D, Deleuran M, et al. Dupilumab provides favorable safety and sustained efficacy for up to 3 years in an open-label study of adults with moderate-to-severe atopic dermatitis. Am J Clin Dermatol. 2020;21(4):567-77.

12. Charman CR, Venn AJ, Ravenscroft JC, Williams HC. Translating Patient-Oriented Eczema Measure (POEM) scores into clinical practice by suggesting severity strata derived using anchor-based methods. Br J Dermatol. 2013;169(6):1326-32.

13. Schram ME, Spuls PI, Leeflang MM, Lindeboom R, Bos JD, Schmitt J. EASI, (objective) SCORAD and POEM for atopic eczema: responsiveness and minimal clinically important difference. Allergy. 2012;67(1):99-106. 
14. Basra MK, Fenech R, Gatt RM, Salek MS, Finlay AY. The Dermatology Life Quality Index 1994-2007: a comprehensive review of validation data and clinical results. Br J Dermatol. 2008;159(5):997-1035.

15. Finlay AY, Khan GK. Dermatology Life Quality Index (DLQI)-a simple practical measure for routine clinical use. Clin Exp Dermatol. 1994;19(3): 210-6.

16. Basra MK, Salek MS, Camilleri L, Sturkey R, Finlay AY. Determining the minimal clinically important difference and responsiveness of the Dermatology Life Quality Index (DLQI): further data. Dermatology. $2015 ; 230(1): 27-33$.

17. EuroQoL. EuroQoL instruments. 2018. https:// euroqol.org/eq-5d-instruments/. Accessed June 2021.
18. Hongbo Y, Thomas CL, Harrison MA, Salek MS, Finlay AY. Translating the science of quality of life into practice: what do dermatology life quality index scores mean? J Invest Dermatol. 2005;125(4): 659-64.

19. Simpson EL, Beck LA, Wu R, et al. Correlation between clinical and patient-reported outcomes in patients with moderate-to-severe atopic dermatitis in a phase 2 clinical trial of dupilumab. Poster presented at 23rd World Congress of Dermatology (WCD); Vancouver; 2015.

20. Kimball AB, Yves P, Stephen $\mathrm{T}$, et al. Interpretation of EASI-75 from a patient perspective-a post hoc analysis from a phase $2 \mathrm{~b}$ trial of dupilumab in adults with moderate-to-severe atopic dermatitis. JAAD. 2017;76(6 Suppl 1):416. 